\title{
Non-axisymmetric oscillations of liquid bridges
}

\author{
By A. SANZ AND J. LOPEZ DIEZ \\ Laboratorio de Aerodinámica, ETSI Aeronáuticos, Universidad Politécnica, \\ 28040 Madrid, Spain
}

(Received 27 November 1987 and in revised form 10 February 1989)

The main characteristics of the non-axisymmetric oscillations of a liquid bridge have been considered : free frequencies, deformation modes and the influence of an outer liquid. Oscillations of this kind do not show stability changes.

The Plateau technique has been used to obtain the resonant frequencies of the bridge when lateral perturbations are imposed. The results obtained are in good agreement with the theoretical ones when the influence of the outer liquid is considered. Moreover, lateral oscillations observed in experiments performed with liquid bridges in space can be explained with this model.

\section{Introduction}

The aim of this paper is to complete the analysis of one of the outstanding mechanical characteristics of a system like the liquid bridge, the determination of the free frequencies, and the related deformation shapes.

The interest which the study of the liquid-bridge configuration has received is reflected in the number of publications on this subject (see review by Meseguer \& Sanz 1985). More recent studies deal with experiments performed in space, aboard Spacelab during the D-1 Mission (Martínez 1987a, 1987b, private communication; Meseguer, Sanz \& López 1986).

A review of the studies on the oscillation modes of a liquid bridge can be found in Sanz (1985). He pointed out the differences between models for capillary jets (or infinite liquid columns) and liquid bridges (of finite length). These differences are caused by the boundary conditions at the disks (anchoring of the interface and zero normal velocity) which are not fulfilled by capillary jet models (Rayleigh 1945; Tomotika 1935; Bauer 1982).

Although the one-dimensional models (Lee 1974; Meseguer 1983; Rivas \& Meseguer 1984) are useful for axisymmetric motions, even of finite amplitude, they cannot be extended to the non-axisymmetric case. In addition, these and the quasione-dimensional models (Entov \& Yarin 1984) are limited to slender columns. Here the model of linear oscillations of Sanz (1985) is extended to include the nonaxisymmetric modes. The presence of these additional degrees of freedom implies a more complicated solution of the problem.

The effect of viscosity on the liquid-bridge dynamics is assumed to be small in the experimental configuration of interest. An estimation criterion is offered in the conclusions.

The main application of the liquid-bridge stability studies concerns its motion in microgravity conditions, where it is surrounded by vacuum or a gas (of much lower density). However, the effect of an outer liquid has been considered to obtain results which can be applied to the configuration appearing in the neutral buoyancy 
simulation technique used to perform experiments on Earth. In the liquid-bridge study this simulation technique has been used to determine the static stability limits (Sanz \& Martínez 1983; Russo \& Steen 1986), to check the results of the onedimensional model concerning the breaking dynamics (Meseguer \& Sanz 1985), and the combined influence of both a small axial gravity and differences in disk diameters (Meseguer et al. 1985), and to study the resonant frequencies of axisymmetric oscillations (Sanz 1985).

\section{General inviscid equations}

Consider a liquid bridge held by surface-tension forces between two parallel coaxial, equal-diameter solid disks, surrounded by an outer liquid, immiscible with the inner one, as shown in figure 1. The assumptions of this model are as follows:

(a) The shape of the interface differs slightly from a cylinder.

(b) Movements inside the liquid bridge and the bath are due to capillary pressure gradients generated by the deformation of the interface.

(c) The densities of both liquids as well as surface tension are uniform and constant.

(d) The viscosity effects are small enough to consider the motion as inviscid.

Under these assumptions the velocity field can be considered to be derived from a velocity potential, $\phi^{j}(r, z, \theta ; t)$, both in the liquid bridge $(j=$ 'i') and in the outer liquid $\left(j={ }^{\prime} o^{\prime}\right)$. Both $\phi^{j}$ satisfy the Laplace equation

$$
\Delta \phi^{j}=0
$$

and the pressure fields $P^{j}$ are related to the velocity potentials through

$$
P^{j}=-\rho^{j} \phi_{t}^{j}-\frac{1}{2} \rho^{j}\left(\nabla \phi^{j}\right)^{2}
$$

where $\rho^{j}$ are the densities of the fluids and $t$ is the time coordinate. The velocity components relative to coordinates $r, z, \theta$, are $U^{j}, W^{j}, V^{j}$ respectively. Lengths are made dimensionless with disk radius, $R$, velocities with $\left(\sigma / \rho^{\mathrm{i}} R\right)^{\frac{1}{2}}$ where $\sigma$ is the interface tension. The other characteristic magnitudes are derived from these two.

Boundary conditions are:

(a) At the solid surfaces

$$
\begin{gathered}
W^{\mathrm{I}}(r, \pm A, \theta, t)=W^{\circ}(r, \pm A, \theta, t)=0, \\
U^{\mathrm{o}}(B, z, \theta, t)=0,
\end{gathered}
$$

where $A=L / 2 R$ is the slenderness of the liquid bridge, $B$ the dimensionless radius of the external cylindrical container (wall position), and $t$ is the dimensionless time.

(b) At the interface, $r=F(z, \theta, t)$, two conditions hold. The first is that the interface is a material surface

$$
F_{t}-U^{j}+W^{j} F_{z}+\frac{1}{r} V^{j} F_{\theta}=0
$$

where subscripts represent derivatives. The second is the normal balance between the capillary pressure and the static pressure jump across the interface (Struik 1957)

$$
P^{1}-P^{o}+\frac{F\left(1+F_{z}^{2}\right)\left(F_{\theta \theta}-F\right)+F F_{z z}\left(F^{2}+F_{\theta}^{2}\right)-2 F_{\theta}\left(F_{\theta}+F F_{z} F_{z \theta}\right)}{\left[F^{2}\left(1+F_{z}^{2}\right)+F_{\theta}^{2}\right]^{\frac{3}{2}}}=0,
$$




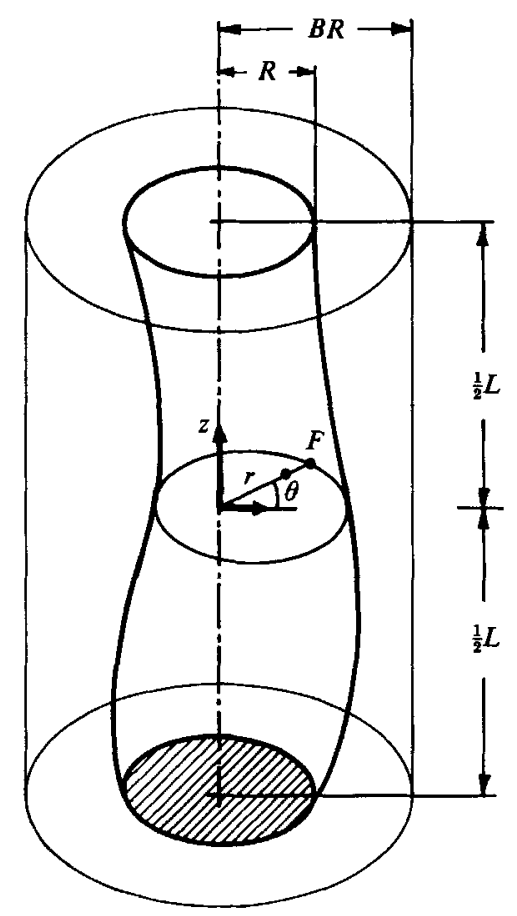

Figure 1. Geometry and coordinate system for the surrounded liquid bridge in the nonaxisymmetric case.

where $P^{j}$ are the static pressures at the interface. The interface itself should fulfil the conditions of anchorage, periodicity and volume preservation, in this case the volume of a cylinder :

$$
F( \pm \Lambda, \theta, t)=1, \quad F(z, \theta, t) \equiv F(z, \theta+2 \pi, t), \quad \frac{1}{2} \int_{-A}^{\Lambda}\left[\int_{0}^{2 \pi} F^{2} \mathrm{~d} \theta\right] \mathrm{d} z=2 \pi \Lambda .
$$

Suitable initial conditions should be added.

\section{Linear analysis}

Let $\epsilon$ be a small parameter which measures the maximum deformation of the initial interface shape with respect to the cylinder. Assuming the dependence on time as $\mathrm{e}^{s t}$, the variables of the problem can be expressed as follows

$$
\left[\phi^{j}, P^{\mathrm{i}}-1, P^{\mathrm{o}}, F-1, U^{j}, V^{j}, W^{j}\right]=\epsilon \mathrm{e}^{s t}\left[\psi^{j}, p^{\mathrm{i}}, p^{\mathrm{o}}, f, u^{j}, v^{j}, w^{j}\right] .
$$

By neglecting higher-order terms, (2.1)-(2.6) reduce to

$$
\begin{gathered}
\Delta \psi^{j}=0 \\
\psi_{z}^{1}(r, \pm \Lambda, \theta)=\psi_{z}^{o}(r, \pm \Lambda, \theta)=0 \\
\psi_{r}^{o}(B, z, \theta)=0
\end{gathered}
$$

The conditions at the interface are imposed at $r=1$, the linearized position of the interface,

$$
\begin{gathered}
s f_{t}=\psi_{r}^{\mathrm{i}}=\psi_{r}^{\mathrm{o}} \\
f_{z z}+f_{\theta \theta}+f=s\left(\psi^{\mathrm{i}}-\rho \psi^{\circ}\right),
\end{gathered}
$$


where $\rho=\rho^{\circ} / \rho^{\mathrm{i}}$. The conditions (2.6) for the interface are

$$
f( \pm \Lambda, \theta)=0, \quad f(z, \theta)=f(z, \theta+2 \pi), \quad \int_{-\Lambda}^{\Lambda} \int_{0}^{2 \pi} f \mathrm{~d} z \mathrm{~d} \theta=0 .
$$

The method followed to solve the problem is similar to that employed in the study of axisymmetric oscillations (Sanz 1985) although the non-axisymmetric motions formulation is somewhat more complex. The axisymmetric motions, although initially retained, will be omitted in the calculations to simplify the presentation. The solutions of the problem which are periodic in the $\theta$-coordinate and satisfy the boundary conditions $(3.3),(3.4)$ and $(3.5)$, are

$$
\begin{aligned}
& \psi^{\mathrm{i}}=a_{00}+\sum_{m=1}^{\infty} a_{m 0} r^{m} \cos m \theta+\sum_{\substack{m=0 \\
n=1}}^{\infty, \infty} a_{m n} I_{m}\left(l_{n} r\right) \cos l_{n}(z+\Lambda) \cos m \theta \\
& \psi^{0}=b_{00}+\sum_{m=1}^{\infty} b_{m 0} r^{m}\left[1+\left(\frac{B}{r}\right)^{2 m}\right] \cos m \theta \\
&+\sum_{\substack{m=0 \\
n=1}}^{\infty, \infty} b_{m n}\left[K_{m}\left(l_{n} r\right)-I_{m}\left(l_{n} r\right) \frac{K_{m}^{\prime}\left(l_{n} B\right)}{I_{m}^{\prime}\left(l_{n} B\right)}\right] \cos l_{n}(z+\Lambda) \cos m \theta \\
& s f=\sum_{m=0}^{\infty}\left\{m a_{m 0}+\sum_{n=1}^{\infty} a_{m n} l_{n} I_{m}^{\prime}\left(l_{n}\right) \cos l_{n}(z+\Lambda)\right\} \cos m \theta
\end{aligned}
$$

where $\quad l_{n}=\frac{n \pi}{2 \Lambda}, \quad b_{m 0}=a_{m 0}\left(1-B^{2 m}\right)^{-1}, \quad b_{m n}=a_{m n}\left(\frac{K_{m}^{\prime}\left(l_{n}\right)}{I_{m}^{\prime}\left(l_{n}\right)}-\frac{K_{m}^{\prime}\left(l_{n} B\right)}{I_{m}^{\prime}\left(l_{n} B\right)}\right)^{-1}$,

$a_{m n}$ are arbitrary constants, $I_{m}(x), K_{m}(x)$ are the $m$-order modified Bessel functions, and primes denote their derivatives with respect to the argument. The solution corresponding to $n=0$, which represents a two-dimensional flow in the plane $r, \theta$, should be explicitly retained. Only a generic term in $m$ of the double summation need be considered since the azimuthal modes are not coupled by the boundary conditions in this problem.

For each mode $m$ there are $n+3$ arbitrary constants as in the axisymmetric case : $n+1$ from the pressure jump $\left(a_{m n}, a_{00}-\rho b_{00}\right)$, and two from the integration of (3.6). On the other hand, there are $n+3$ relationships: three conditions (the first and last in (3.7)), and $n$ equations coming from the identification of $f$ (in harmonic components) in expression (3.9) and the integration of (3.6). The algebraic homogeneous system obtained will have solutions only for values of the parameters involved $(\Lambda, s, m, \rho)$ that satisfy some secular equations.

The integration of (3.6) by using (3.8) valued at $r=1$ is the combination of the homogeneous, $f_{\mathrm{h}}$, and particular, $f_{\mathrm{p}}$, solutions, $f=f_{\mathrm{h}}+f_{\mathrm{p}}$,

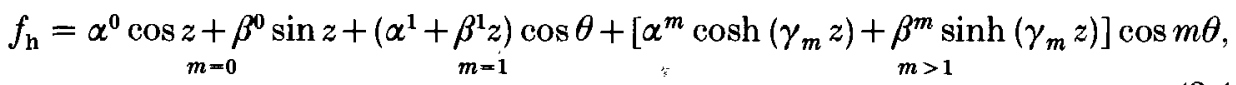

$$
\begin{aligned}
& s^{-1} f_{p}=a_{00}-\rho b_{00}+\frac{1}{2} a_{10} S_{10} z^{2} \cos \theta+a_{m 0} \underbrace{}_{\substack{m 0 \\
m>1, n=0}} S_{\substack{m 0 \\
m=0}}^{-1} \cos m \theta \\
& +\sum_{n=1}^{\infty} a_{m n} I_{m} S_{m n} k_{m n}^{-1} \cos l_{n}(z+\Lambda) \cos m \theta
\end{aligned}
$$

In these expressions $S_{m n}$ is defined in (A 2), $\gamma_{m}=m^{2}-1$ and $k_{m n}=-\gamma_{m}-l_{n}^{2}$ (if both $n \neq 0$ and $m \neq 1$ ). The argument of $I_{m}\left(l_{n}\right)$ has been omitted for brevity. The 
axisymmetric case $(m=0)$ has been already treated in Sanz (1985), and so it is not considered here. Related terms $\left(a_{00}-\rho b_{00}, \sin z, \cos z, a_{0 n}\right)$ and the volume preservation condition in (3.7) (which is automatically fulfilled if $m>0$ ) are no longer retained.

The identification of (3.9) and (3.11), using the expansions of $z, z^{2}, \cosh \left(\gamma_{m} z\right)$ and $\sinh \left(\gamma_{m} z\right)$ in cosine series shown in the Appendix, gives $a_{m n}$ as a function of $\alpha^{m}$,

$$
a_{m 0}=\frac{s \alpha^{m} C_{m 0}}{m\left[1-\left(s / s_{m 0}\right)^{2}\right]}, \quad a_{m n}=\frac{s \alpha^{m} C_{m n}}{l_{n} I_{m}^{\prime}\left[1-\left(s / s_{m n}\right)^{2}\right]},
$$

where the coefficients $C_{m n}$ and $s_{m n}$ are defined in the Appendix. The term $\alpha^{m}$ (associated to the symmetric deformation modes $n=2 p$ ) should be replaced by $\beta^{m}$ if $n=2 p-1$ (antisymmetric modes). The first condition in (3.7) states that both the symmetric and antisymmetric part of $f$ should vanish for $z= \pm A$. Substituting (3.11) in (3.7) the following conditions are obtained

$$
\begin{aligned}
& n=2 p \quad\left\{\begin{array}{ll}
m=1 & S_{m 0}^{-1}+\frac{1}{3} s^{2} A^{2}-2 s^{4} H_{m}=0, \\
m \geqslant 2 & \frac{\Lambda}{\gamma_{m} t h\left(\gamma_{m} A\right)}+\frac{s^{2}}{\gamma_{m}^{2}\left(s_{m 0}^{2}-s^{2}\right)}-2 s^{2} H_{m}=0,
\end{array}\right\} \\
& n=2 p-1\left\{\begin{array}{ll}
m=1 & \Lambda^{2}-2 s^{2} H_{m}=0, \\
m \geqslant 2 & \frac{\Lambda}{\gamma_{m}} t h\left(\gamma_{m} \Lambda\right)-2 s^{2} H_{m}=0,
\end{array}\right\}
\end{aligned}
$$

where

$$
H_{m}=\sum_{n} \frac{1}{k_{m n}\left(s_{m n}^{2}-s^{2}\right)}
$$

in order that non-trivial values of $\alpha^{m}$ or $\beta^{m}$ could arise. For each value of $A$ these secular equations define the allowed values of $s$, associated to one determined mode of oscillation.

An alternative form of (3.13) can be obtained from the first condition in (3.7) written in terms of $f$ given by (3.9) with the aid of (3.12), that is

$$
\sum_{n=0}^{\infty} \frac{C_{m n}}{1-\left(s / s_{m n}\right)^{2}}=0
$$

which can be shown to be equivalent to (3.13) for every value of $m$. Expression (3.14), although more compact, has a slower convergence rate than (3.13).

Equations (A 3) and (3.13) show that these symmetric and antisymmetric modes are quite different. Whereas antisymmetric modes are essentially three-dimensional, the symmetric modes are a combination of plane $(n=0)$ and three-dimensional $(n=2 p)$ motions. In fact, if the component $a_{10}$ is not considered in (3.8) then $C_{12 p}=0$, and thus symmetric modes in $m=1$ will not appear.

In (3.12) $s=s_{m n}$ corresponds to the values given by the infinite column model (Bauer 1982), $n=N$ being the number of half-waves of the deformation contained in the same length of a liquid bridge, $2 A$. However, in the case of a finite column, the values of $s$ that satisfy the secular equation are different from $s_{m n}$ because one single mode as $\cos l_{n}(z+A)$ cannot satisfy simultaneously both boundary conditions at the disks. 

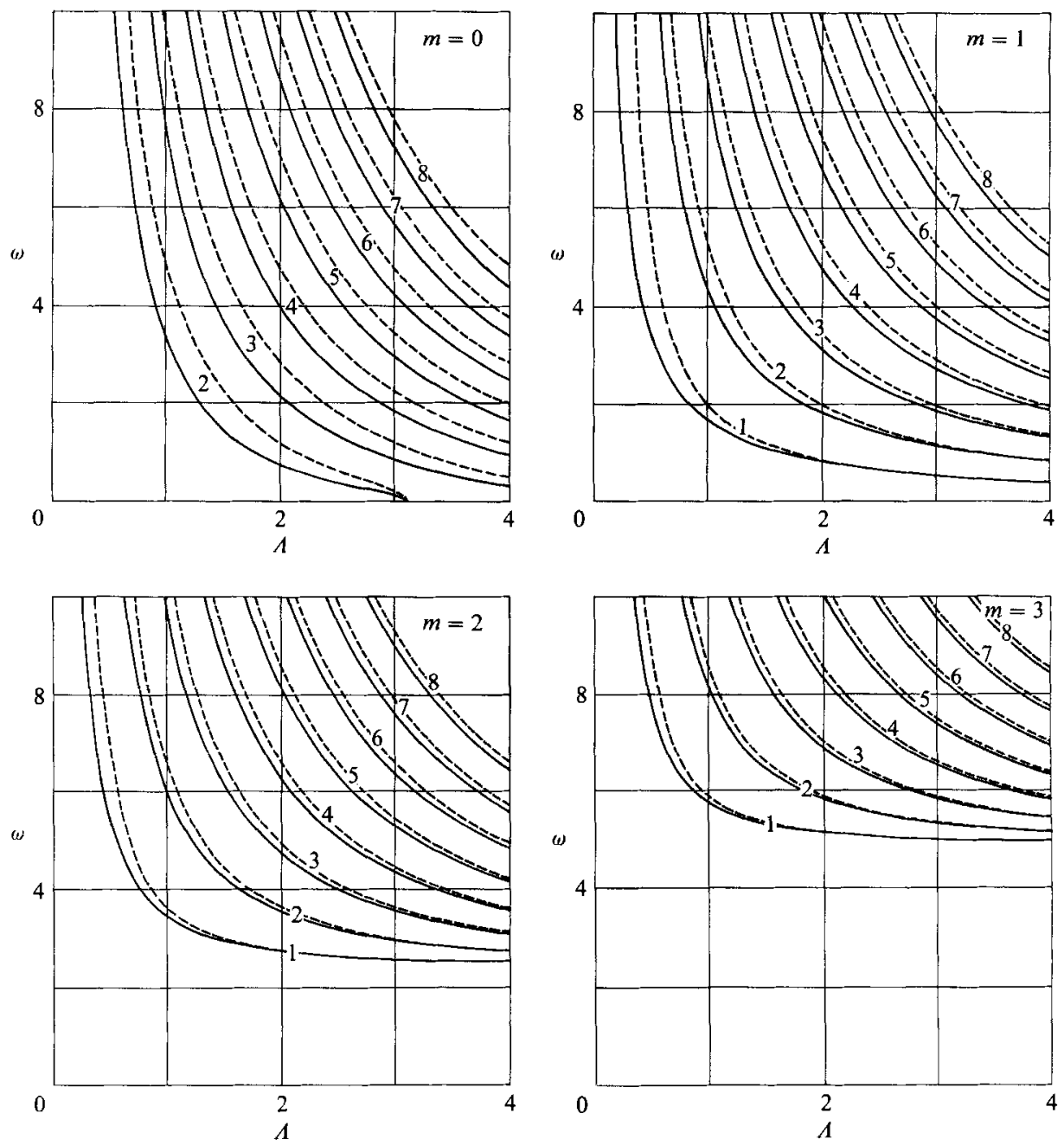

Figure 2. Dimensionless pulsation, $\omega$, versus slenderness, $\Lambda$, of the oscillation motion of a nonsurrounded liquid bridge, for several azimuthal $(m)$ and axial $(N)$ modes. Figures indicate the value of $N$ which is both the order of the root of (3.13) and the number of half-waves of the axial interface deformation. Solid (dashed) lines: finite (infinite, Bauer 1982) column model.

\section{Theoretical results}

In a similar way to that followed in the axisymmetric case (Sanz 1985), it can be shown that the values of $s$ (complex in general) which satisfy (3.13) are such that $s^{2}$ is a real number; $s= \pm \mu$ and $s= \pm \mathrm{i} \omega$ represent divergent or oscillatory motions, respectively ( $\mu$ is the dimensionless growth factor and $\omega$ the dimensionless pulsation). This fact greatly reduces the searching for the roots of (3.13). For each azimuthal mode, and each value of $\Lambda$, the roots $s_{N}$ have been placed in descending order and denoted $s_{N}^{2}=q_{m N}$

$$
q_{m 1}>q_{m 2}>\ldots>q_{m N}>\ldots .
$$

The ordered sequence begins with $N=1$ except if $m=0$ (axisymmetric modes) in which $N=2$ for volume preservation.

The results obtained for the first values of $m$ and $N$ are shown in figure 2 . The 


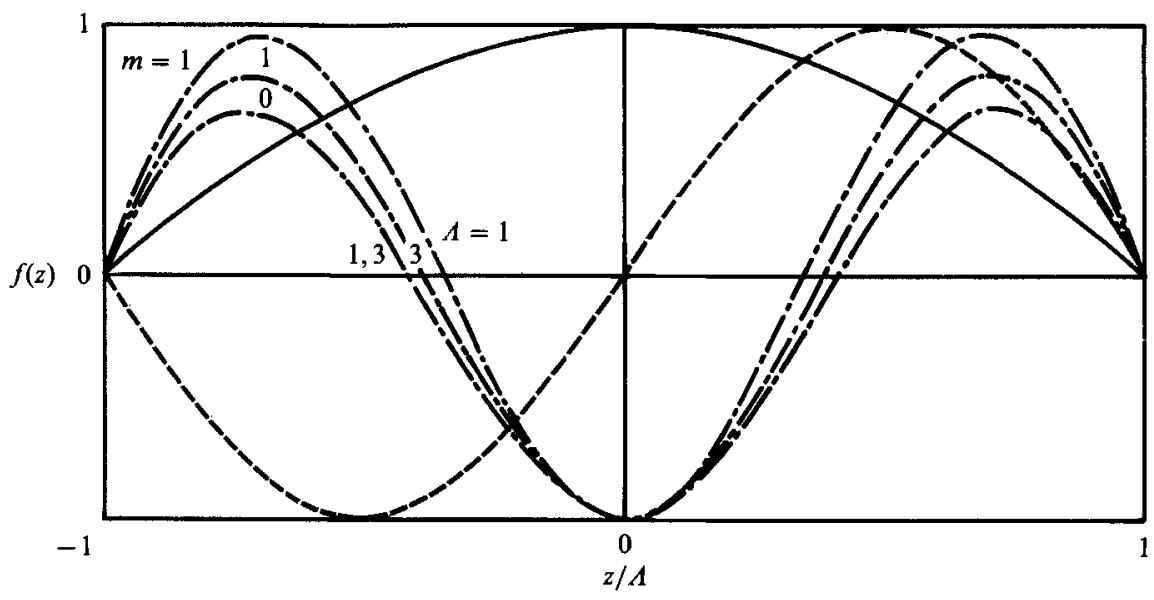

Figure 3. Interface deformations (from cylindrical shape) of non-surrounded liquid bridges and surrounded by an isodense infinite bath for several azimuthal $m$ and axial $N$ oscillation modes. $A$ : slenderness of the bridge. Solid (dashed) lines: $N=1(2)$ with $m=0,1,2,3$ and $A=1,3$. Dotdashed lines: $N=3$, figures indicate the values of $m$ and $A$.

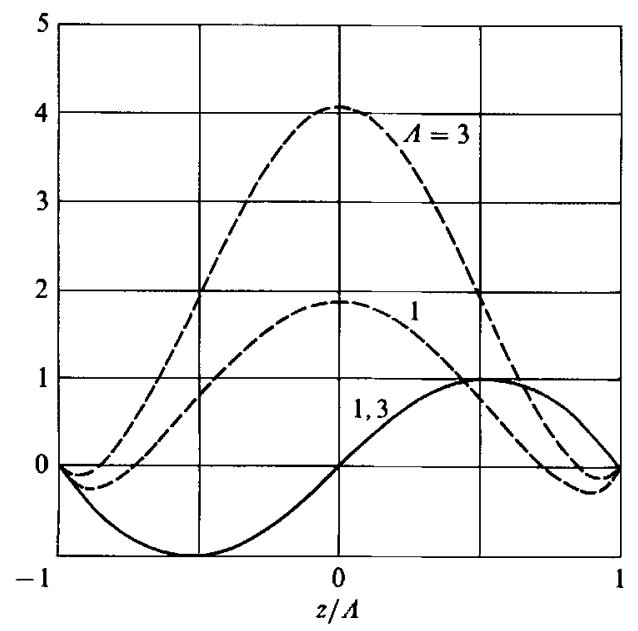

Figure 4. Variation with the axial coordinate, $z$, of the velocity components $u$ (solid line) and $w$ (dashed line) at the interface $(r=1)$ of a liquid bridge, for the axisymmetric mode $(m=0)$ and the first axial mode ( $N=2$ in this case). Figures indicate the slenderness of the bridge, $\Lambda$.

values derived from this model are interleaved with those given by the infinite column model. The infinite column results $q_{m N}^{*}=s_{m N}^{2}$ help with the computations of the true $q_{m N}$, being used as an initial value in the iterative (Newton-Raphson) method employed.

The difference between results from both models reflects the influence of the boundary conditions at the disks. As $m$ increases, results from both models become closer to each other. The larger influence of the disk in the axisymmetric modes is due to the restriction of the axial flow as the net flow across every $r, \theta$ section is not nil except at the disks. In contrast, this net flow is zero across every section in the nonaxisymmetric oscillations, so that disks effects are not so important. Furthermore, as $m$ increases the flow inside the $(r, \theta)$-plane grows and that inside the $(r, z)$-plane decreases. 
(a)

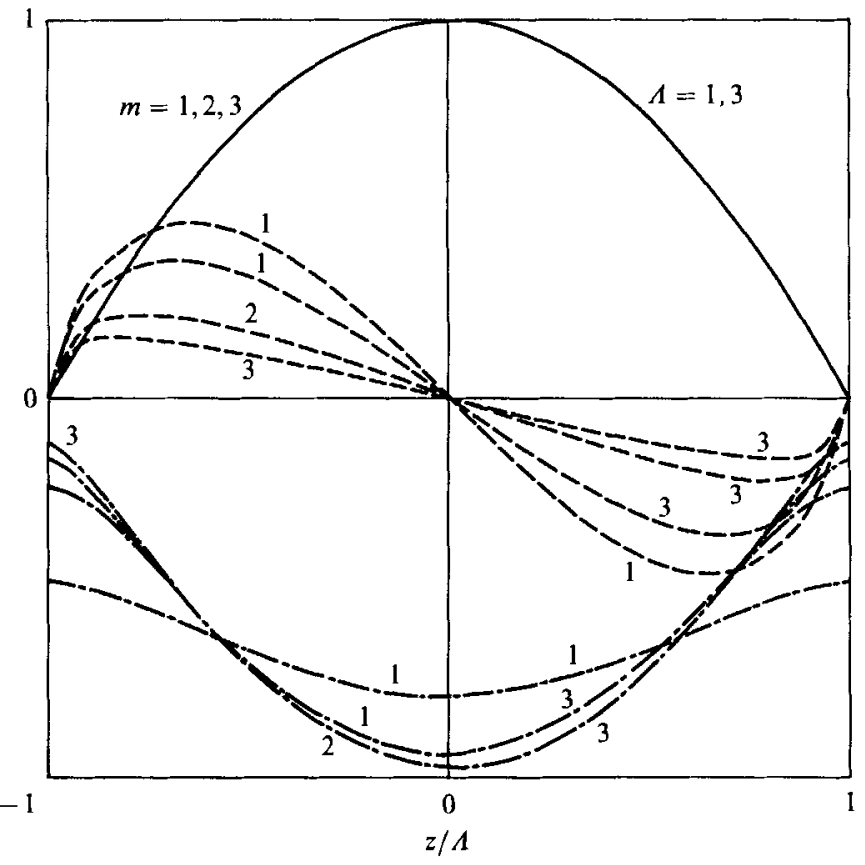

(b)

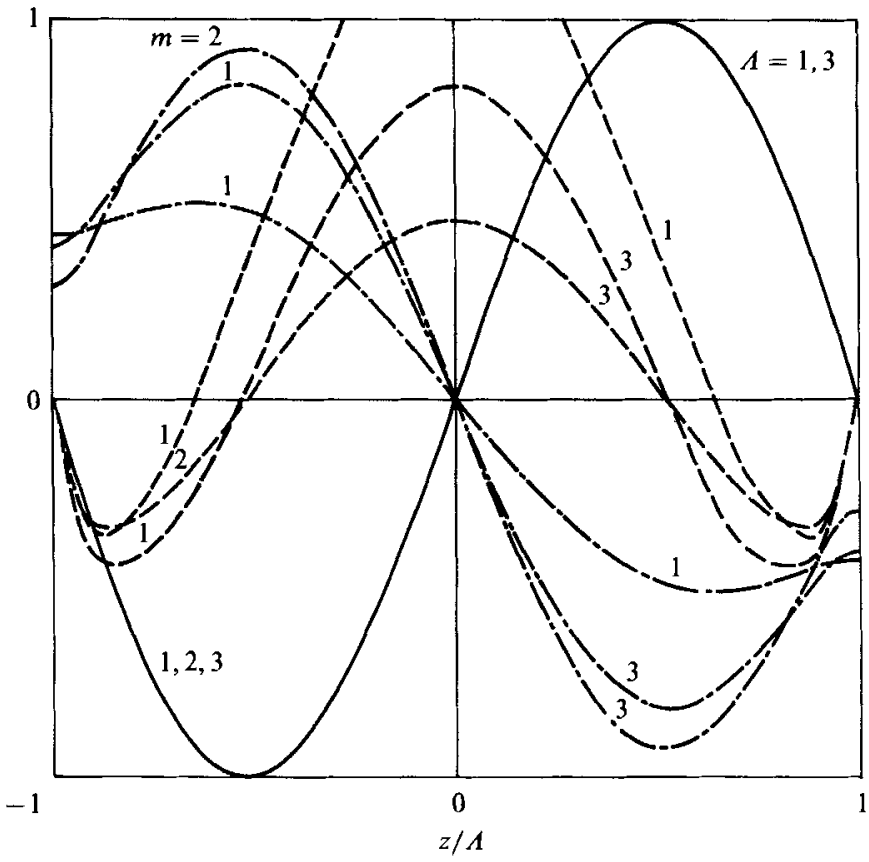

Figure 5. Variation with the axial coordinate, $z$, of the velocity components $u, w, v$ (solid, dashed and dot-dashed lines, respectively) at the interface $(r=1)$ of a liquid bridge for several azimuthal $(m)$ and axial $(N)$ modes. $(a) N=1,(b) N=2 . A$ stands for the slenderness of the bridge. 
0

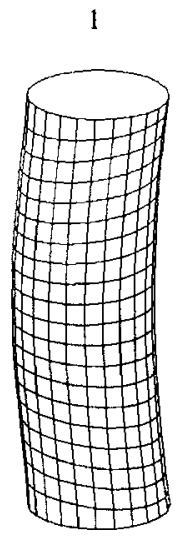

$m$

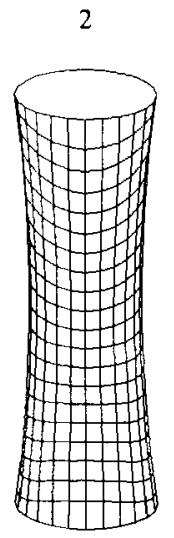

3
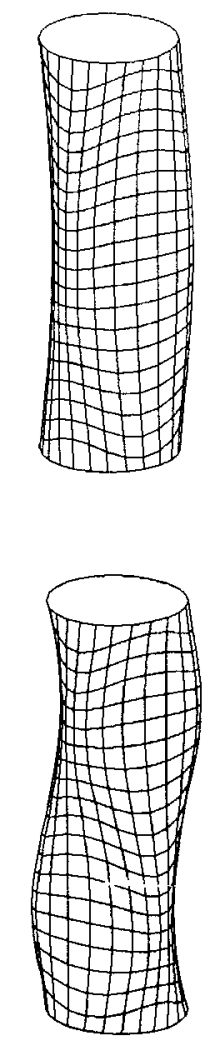
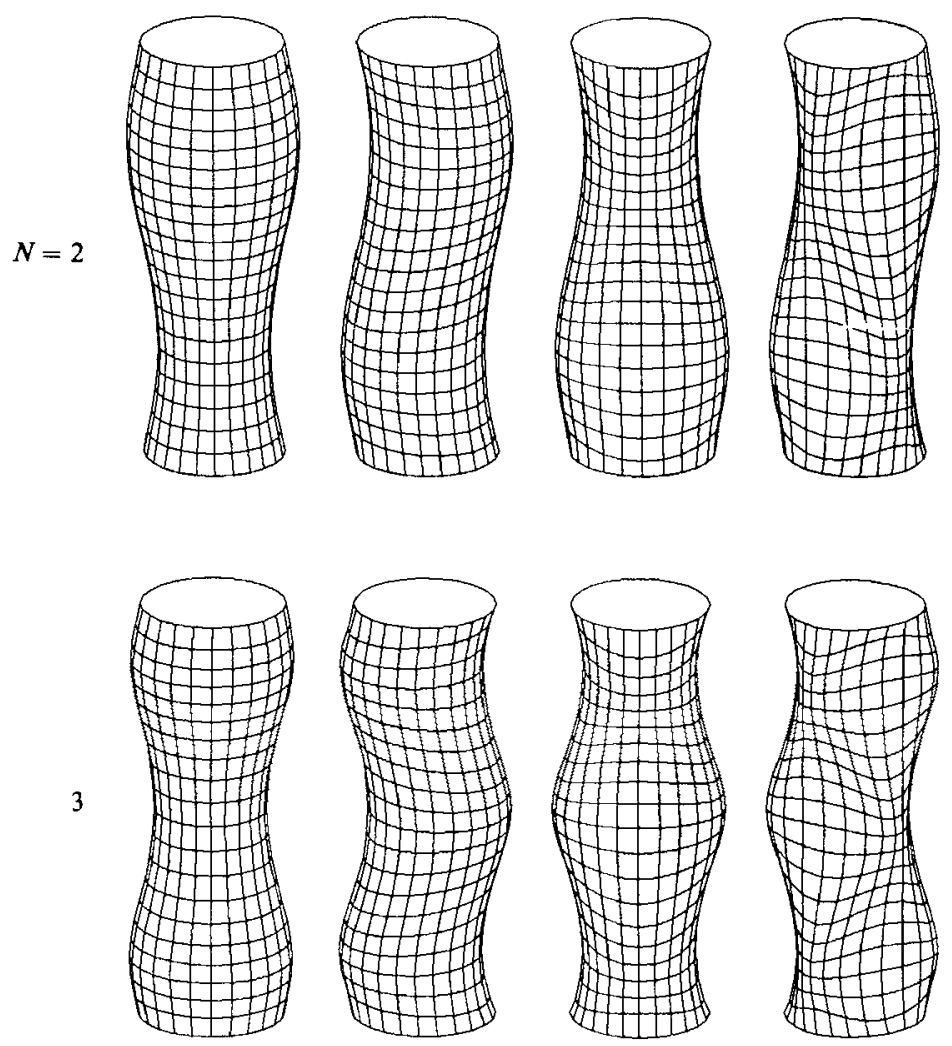

Figure 6. Oscillation modes of the liquid bridge. Figures indicate the azimuthal $(m)$ and axial $(N)$ modes.

The non-axisymmetric modes do not show stability changes because for $s=0$ there is not a value of $A$ (except $A=0$ ) which satisfies (3.13). Thus, $q_{m N}<0$ always, so only stable oscillatory motions exist, which is the same result obtained for finite and infinite columns (Rayleigh 1945; Martínez 1978; Vega \& Perales 1983). Figure 2 also shows that the threshold to excite an azimuthal mode $m$ increases as $m$ increases. 
The interface deformation related to an axial mode $N$ can be obtained by using (3.9) and (3.12) which yields

$$
f=\alpha^{m} \sum_{n=0}^{\infty} \frac{C_{m n}}{1-q_{m N} / s_{m n}^{2}} \cos l_{n}(z+\Lambda) \cos m \theta,
$$

where the summation is made over the odd or even $n$ depending upon the parity of $N$. Equation (4.2) is extended to the case $m=0$ by defining the $C_{0 n}$ coefficients as shown in the Appendix.

The variation with $z$ of the interface deformation in the cases $m=0$ to 3 and $N=1$ to 3 are shown in figure 3 . The deformations of the modes $N=1$ and $N=2$ almost coincide regardless the values of $m$ and $\Lambda$, and closely reproduce the functions $\cos (\pi z /(2 \Lambda))$ and $\sin (\pi z / \Lambda)$, respectively. The influence of $m$ and $\Lambda$ first appears at $N=3$.

Concerning the velocity field, the axial variation of the three velocity components at the interface $(r=1)$ is shown in figures 4 and 5 , for the first values of $m$ and $N$. For $m=0$ axial velocity increases as $A$ increases (whereas the opposite occurs for $m>0)$ and it decreases as $m$ increases, which can be explained in terms of the net flow across each $r, \theta$ section. Whereas for $m=0, w$ is larger than $u$, the opposite occurs for $N=1(m>0)$, and for $N>1, u$ and $w$ become of the same order.

To clarify the deformation modes the shape of the interface has been obtained by deformation of an initially cylindrical rectangular net of equally spaced nodes, placed at the interface, as shown in figure 6 . The nodes are moved according to the velocity vector (with the $w$-component trimmed) at the initial position.

Concerning the influence of the outer liquid no new features appear with respect to the axisymmetric case (Sanz 1985). The influence is concentrated in the reduction of the free frequencies. In $\$ 5.2$ the free frequencies are shown for the case of an isodense outer liquid and $m=1$ which is the simplest experimental configuration to check the theoretical results.

\section{Experimental study}

The main experimental results already performed by using the neutral buoyancy technique concern the dynamics of axisymmetric liquid bridges. However, the need for an interpretation of the experiments carried out in space, on board Spacelab, during the D-1 Mission (Martínez 1987a, 1987b, private communication) and, of course, its own interest have encouraged the completion of the theoretical study of non-axisymmetric motions and the performance of appropriate experiments to validate its results.

One of the main features of the liquid bridge as a dynamic system is the existence of free frequencies, which were selected as the object of the experimental study.

The method followed to obtain the free frequencies consisted of measuring the transfer function of the response of the interface deformation to the lateral oscillation of one of the supporting disks (Fowle, Wang \& Strong 1979; Elagin, Lebedev \& Tsemelev 1982; Bisch, Lasek \& Rodot 1982; Sanz 1985). If viscous effects can be neglected, free frequencies should coincide with the resonant frequencies of the forced system at which the amplitude of the deformation reaches a maximum. Owing to the difficulties in producing perturbations which excited higher modes, only the mode $m=1$ was tried, as it could be excited by the lateral oscillation of one of the supporting disks. 


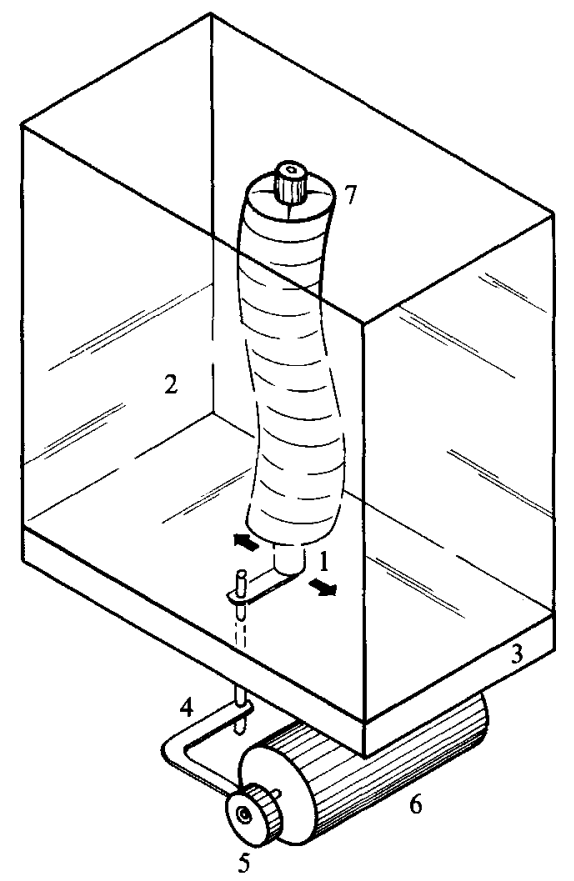

Fiaure 7. Experimental arrangement: 1, excitation disk; 2, bath; 3, tank; 4, oscillation lever; 5 , eccentric; 6, variable speed motor; 7 , upper (and feeding) disk.

\subsection{Apparatus description}

The main characteristics of the apparatus, and the technique can be found in Martínez \& Rivas (1982) and in Sanz (1985). The liquid bridge was formed between two parallel and (if no excitation is imposed) coaxial disks of $2.62 \times 10^{-2} \mathrm{~m}$ diameter and having sharp edges, to enhance the anchorage of the interface.

The liquid for the bridge was a slightly dyed dimethyl silicone oil having a viscosity of $20 \times 10^{-6} \mathrm{~m}^{2} \mathrm{~s}^{-1}$ (Rhone-Poulenc 1978). The bridge was surrounded by a $1: 2$ methanol-water mixture (called bath), whose density was trimmed with the aid of the liquid-bridge deformations (Sanz \& Martínez 1983) to match precisely that of the oil $\left(\rho^{0}=\rho^{1}=945 \mathrm{~kg} \mathrm{~m}^{-3}\right)$. The interfacial tension was measured as $\sigma=(9.7 \pm 0.5) \times 10^{-3} \mathrm{~N} \mathrm{~m}^{-1}$ by using the procedure indicated in Sanz (1985), in the same temperature range as the experiments. The bath and the liquid bridge were contained in a tank of $140 \mathrm{~mm} \times 80 \mathrm{~mm}$ sides and $140 \mathrm{~mm}$ height with glass walls, as shown in figure 7 . Although the theoretical model considers a cylindrical tank, it can be shown that the shape of the tank only affects the resonance frequency when the wall distance, $B$, is close to 1 . In the direction of the lateral motion this distance is $B \approx 5$, so that the influence of the shape of the tank can be neglected.

The lower disk in figure 7 is attached to an axis parallel to the axis of the unperturbed bridge, but placed $3 \times 10^{-2} \mathrm{~m}$ apart. For rotations of small angle the displacement of the disk can be considered as rectilinear. An observer placed in front of the tank sees the disk performing the lateral displacement required. The oscillatory motion of this axis is produced by a variable speed controlled motor and an eccentric of variable amplitude (1-6.5 mm peak to peak). The axis of the motor is provided with an optical encoder which allows the detection of the angular position of the eccentric. A photographic camera can be synchronized at the required phase 


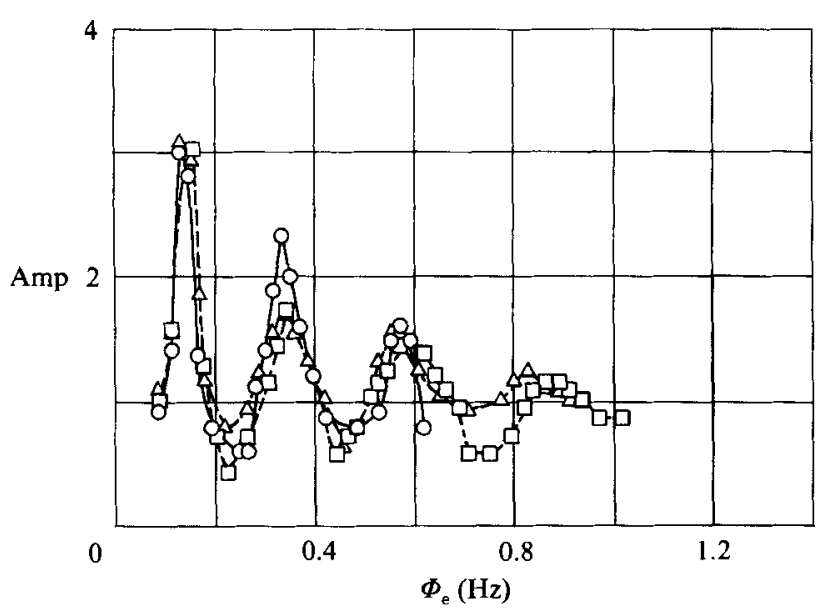

Figure 8. Variation of the amplification, Amp (the ratio of maximum interface deformation, $2 R f_{\max }$, to the excitation amplitude, $A_{\mathrm{e}}$ ), with the excitation frequency, $\Phi_{\mathrm{e}}$, of a liquid bridge of slenderness $A=2.6$, surrounded by a density-matched liquid for several values of $A_{\mathrm{e}}: 0,2.5 \mathrm{~mm}$; $\square, 3.5 \mathrm{~mm} ; \triangle, 6.5 \mathrm{~mm}$.

of the cycle to determine the angular position of the maximum deformation. Temperature measurements were made during experiments, and readings were in the range $26-36^{\circ} \mathrm{C}$.

The visualization system consisted of background illumination, a video camera, a monitor and a video tape recorder. A background grid provided diffuse illumination and reference lines for deformations measurements. The liquid bridge is enlarged some 10 times by the system so that a segment of the bridge (the neighbourhood of the lower disk) appears in the screen. This procedure allows one to measure the interface deformations directly with an error of some $\pm 0.3 \mathrm{~mm}$. Every experiment was recorded for later analysis of the oscillation cycle and repetition of measurements. The optical effect at the interface helped to visualize interface deformations.

\subsection{Methods and results}

The determination of the transfer function at a given $A$ was performed using the frequency sweep method: the maximum amplitude of the half-wave of the interface deformation nearest to the lower disk, $2 f_{\text {max }}$, was measured in several cycles of oscillation at a given excitation frequency, $\Phi_{\mathrm{e}}$, and excitation amplitude, $A_{\mathrm{e}}$, then the frequency was slightly increased and new measurements were performed till the frequency range $(0,1.4 \mathrm{~Hz})$ was covered.

The error in the excitation frequency is $\pm 0.01 \mathrm{~Hz}$, although the error in the resonant frequency, typically $\pm 0.02 \mathrm{~Hz}$, depends on the peak width. One characteristic transfer function, amplification, $A m p=2 R f_{\max } / A_{\mathrm{e}}$, versus excitation frequency, $\Phi_{\mathrm{e}}$, is shown in figure 8 . The highest peak occurs at the first resonant frequency and the height of the peaks decreases as $N$ increases because viscous effects increase with $N$, as shown in the conclusions. The first two resonant shapes are easily detected, but to detect the higher ones, large excitation amplitudes should be applied. The amplifications measured in most of the configurations for the first three modes were roughly 3,2 and 1.5 , respectively (table 1 ), regardless of the value of the slenderness. The first four resonant shapes are shown in figure $9(a)$.

In the case of axisymmetric oscillations, at least in low-damped systems, there 


\begin{tabular}{|c|c|c|c|c|c|c|c|}
\hline \multirow[b]{2}{*}{$\Lambda$} & \multicolumn{4}{|c|}{$\Phi_{\mathrm{R}}(\mathrm{Amp})$} & \multirow[b]{2}{*}{$A_{\mathrm{e}}$} & \multirow[b]{2}{*}{$\Delta T$} & \multirow[b]{2}{*}{$D$} \\
\hline & $N=1$ & $N=2$ & $N=3$ & $N=4$ & & & \\
\hline 1.0 & $0.44(3.4)$ & $1.15(1.8)$ & - & -- & 2.5 & $27.3-28.6$ & 25 \\
\hline 1.0 & $0.44(4.0)$ & $1.15(2.1)$ & - & - & 1.0 & $29.7-31.3$ & 30 \\
\hline 1.0 & $0.38(3.3)$ & $1.00(1.8)$ & - & - & 1.0 & $26.2-27.9$ & 30 \\
\hline 1.0 & $0.43(3.4)$ & $1.15(1.8)$ & - & -- & 1.0 & $29.3-31.4$ & 35 \\
\hline 1.2 & $0.33(3.5)$ & $0.83(2.0)$ & - & - & 1.0 & $34.6-35.7$ & 30 \\
\hline 1.4 & $0.25(3.5)$ & $0.62(2.0)$ & - & - & 1.0 & $28.4-29.5$ & 20 \\
\hline 1.4 & $0.27(3.3)$ & $0.67(1.8)$ & - & - & 1.0 & $28.7-30.2$ & 25 \\
\hline 1.4 & $0.28(3.2)$ & $0.71(1.9)$ & - & - & 1.0 & $33.2-34.4$ & 30 \\
\hline 1.5 & $0.25(4.0)$ & $0.62(2.0)$ & $1.11(1.5)$ & - & 1.0 & $34.0-36.4$ & 40 \\
\hline 1.6 & $0.24(3.2)$ & $0.58(1.8)$ & - & - & 1.0 & $27.0-30.0$ & 35 \\
\hline 1.6 & $0.25(3.3)$ & $0.60(2.0)$ & 一 & - & 1.0 & $31.8-33.4$ & 30 \\
\hline 1.8 & $0.22(3.2)$ & $0.49(2.0)$ & - & - & 1.0 & $29.0-30.0$ & 30 \\
\hline 1.8 & $0.22(2.7)$ & $0.51(2.0)$ & $0.87(1.5)$ & - & 1.0 & $28.0-29.6$ & 40 \\
\hline 2.0 & $0.20(3.0)$ & $0.49(1.8)$ & $0.84(1.5)$ & - & 1.0 & $26.5-27.5$ & 45 \\
\hline 2.0 & $0.20(3.8)$ & $0.48(2.2)$ & $0.82(1.4)$ & - & 2.5 & $33.3-35.0$ & 55 \\
\hline 2.0 & $0.20(3.7)$ & $0.44(2.3)$ & $0.73(1.5)$ & - & 1.0 & $34.0-35.7$ & 45 \\
\hline 2.2 & $0.18(2.7)$ & $0.42(2.1)$ & - & - & 1.0 & $29.8-31.7$ & 25 \\
\hline 2.2 & $0.18(3.0)$ & $0.41(2.0)$ & - & - & 1.0 & $28.2-29.2$ & 30 \\
\hline 2.6 & $0.14(3.0)$ & $0.34(2.3)$ & $0.58(1.6)$ & - & 2.5 & $34.6-35.8$ & 35 \\
\hline 2.6 & $0.15(3.0)$ & $0.35(1.7)$ & $0.59(1.4)$ & $0.88(1.2)$ & 3.5 & $33.4-36.5$ & 62 \\
\hline 2.6 & $0.14(3.1)$ & $0.35(1.7)$ & $0.56(1.5)$ & $0.83(1.2)$ & 6.5 & $34.5-35.0$ & 60 \\
\hline
\end{tabular}

TABLE 1. Slenderness $A$, resonant frequency $\Phi_{\mathrm{R}}(\mathrm{Hz})$, amplification Amp, oscillation mode $N$, peak to peak excitation amplitude $A_{\mathrm{e}}(\mathrm{mm})$, bath temperature $T\left({ }^{\circ} \mathrm{C}\right)$, duration $D(\mathrm{~min})$. The error in $A$ is \pm 0.04 ; in $\Phi_{\mathrm{R}}$ is \pm 0.02 ; in amplification is $\pm 0.3, \pm 0.12$ and \pm 0.05 (corresponding to $A_{\mathrm{e}}=1,2.5$ and $6.5 \mathrm{~mm}$ respectively).

exists a $\frac{1}{2} \pi$ delay between the excitation and the response. The same occurs in the non-axisymmetric case; successive shapes of an oscillation cycle are shown in figure $9(b)$.

The transfer functions have been determined for several values of the slenderness, A. From them, the resonant frequencies, $\Phi_{\mathrm{R}}$, have been deduced. A summary of the results obtained can be found in table 1 . According to the characteristic magnitudes introduced in $\$ 2$, the characteristic time is $\left(\sigma /\left(\rho^{i} R^{3}\right)\right)^{\frac{1}{2}}$. So that the dimensional frequency is related to the dimensionless pulsation, $\omega$, by the expression

$$
\omega=2 \pi \Phi\left(\frac{\rho^{\mathrm{i}} R^{3}}{\sigma}\right)^{\frac{1}{2}} .
$$

The experimental and theoretical results, in dimensionless form, have been compared in figure 10. The influence on resonant frequencies of the excitation amplitude is very small, at least in the range of excitations $\left(0.04<A_{\mathrm{e}} /(2 R)<0.25\right)$ and deformations $\left(0.1<f_{\max }<0.8\right)$ tried, so that the theoretical model remains valid even though it is formally valid only for infinitesimal deformations.

Another data source of great interest is the experimental sequence performed on board Spacelab during the D-1 Mission (Martínez $1987 \mathrm{~b}$, private communication). In this case the liquid bridge (silicone oil of $5 \times 10^{-6} \mathrm{~m}^{2} \mathrm{~s}^{-1}$ viscosity, $\rho^{\mathrm{i}}=920 \mathrm{~kg} \mathrm{~m}^{-3}$, $\sigma=20 \times 10^{-3} \mathrm{~N} \mathrm{~m}^{-1}$ ) supported between $35 \mathrm{~mm}$ diameter disks was surrounded by 
(a)
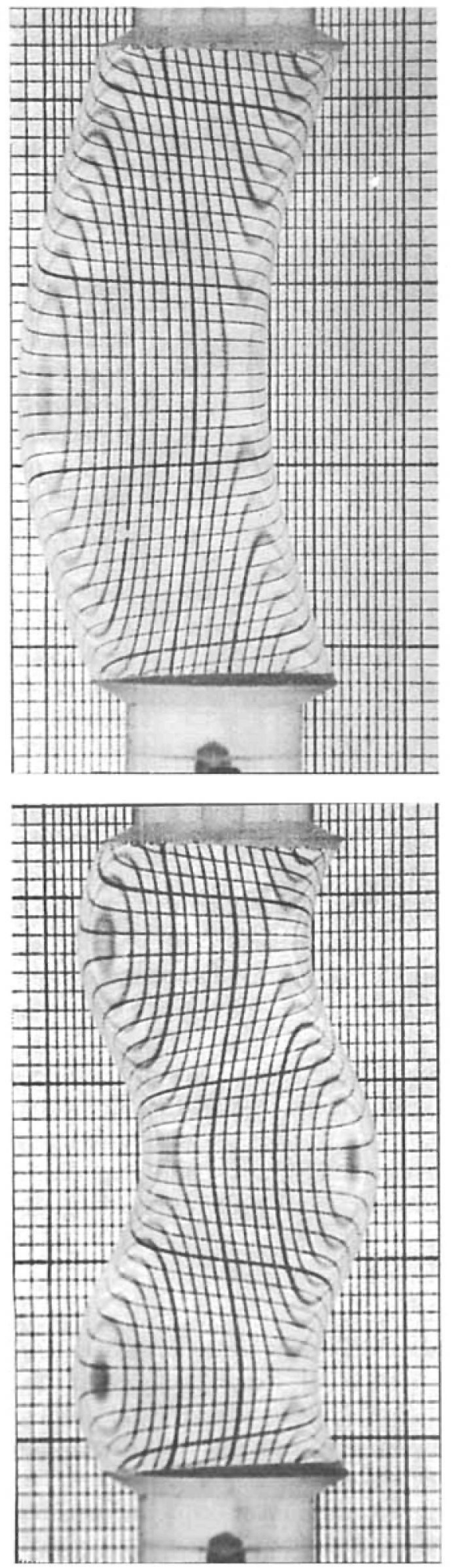
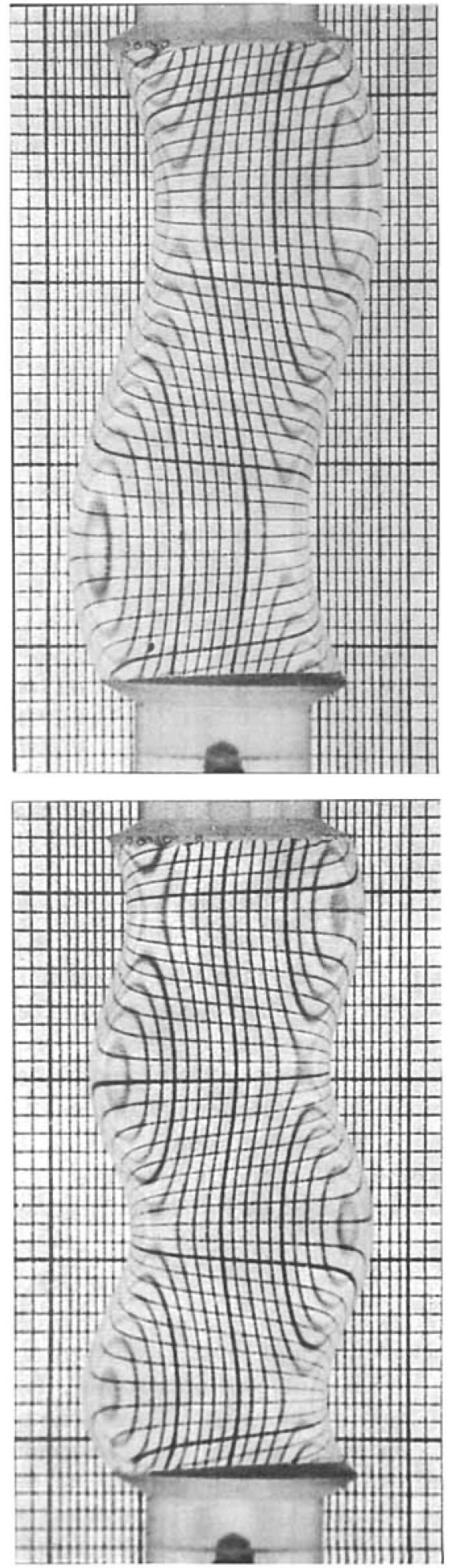

Figure $9(a)$. For caption see facing page. 
(b)
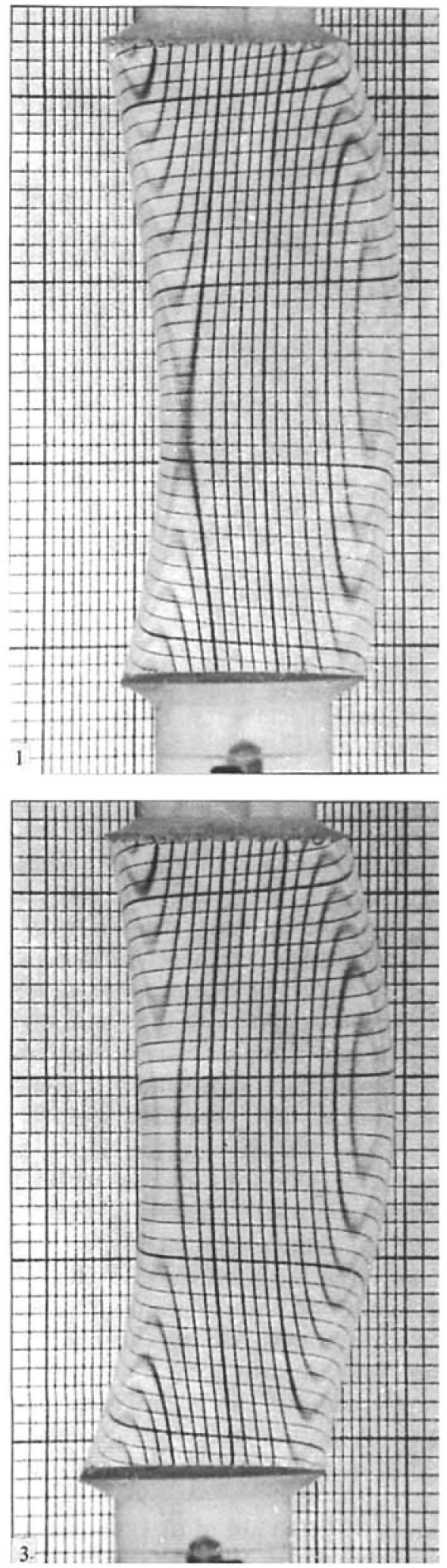
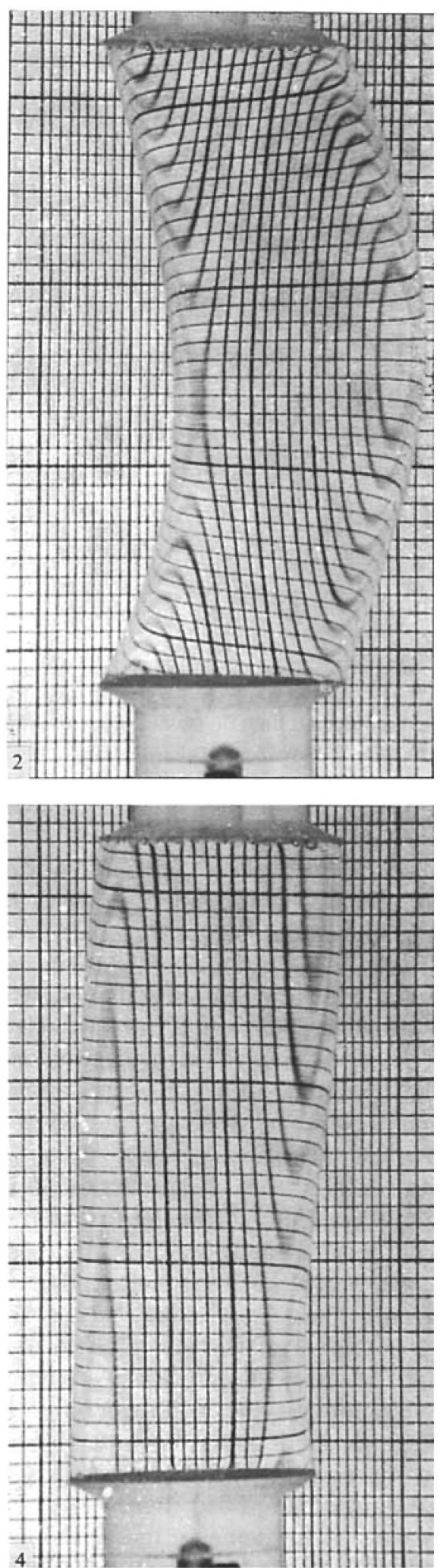

Figure 9. (a) The first four axial modes of lateral oscillations $(m=1)$ at the resonant frequencies of the surrounded liquid bridge taken in the experiment reported in the last row of table 1. (b) Successive shapes of an oscillation cycle at the first mode resonant frequency in the experiment mentioned above. Figures denote the phase between the central position of the disk and the position at the shot : $1,-50^{\circ} ; 2,-10^{\circ} ; 3,50^{\circ} ; 4,90^{\circ}$. 


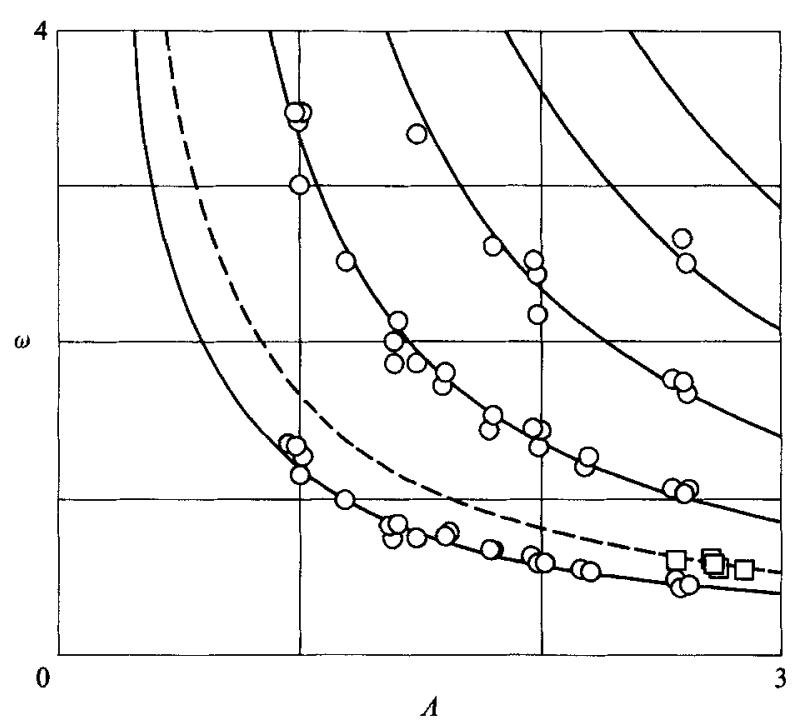

FiguRe 10. Dimensionless pulsation, $\omega$, versus slenderness, $A$, of a liquid bridge. Theoretical results on the lateral oscillation $(m=1)$ : solid lines, first five axial modes of a density-matched liquid bridge; dashed line, first axial mode of a non-surrounded liquid bridge. Experimental results made dimensionless with (5.1) : (O, table 1$)$, neutral buoyancy; $(\square$, table 2$)$ Spacelab-D1. Observe that the characteristic time is different in each case.

air so that $\rho \approx 10^{-3}$. There were no lateral excitations imposed by the experimenter, and the response obtained was due to the residual acceleration of the spacecraft ( $g$-jitter). The study performed to analyse the axisymmetric projection of the motion shows (Meseguer \& Sanz 1987) that the axial component of the acceleration could be characterized as a white noise (constant spectral density) superimposed on a mean value slowly changing with time, at least in the low-frequency range analysed and during the periods of experimentation in which there were no manoeuvres and the perturbations due to crew activities were reduced to a minimum. The same assessment could be done for the lateral component. Following the logic in that reference, the random process theory could help to explain the liquid-bridge behaviour. This theory shows (Bendat \& Piersol 1971) that for processes with a white noise input the squared transfer function is proportional to the spectral density of the response. If the system is slowly damped it would behave as a narrow band filter, only transferring the frequencies closer to the resonance, and the response should exhibit only these frequencies. In fact this selection of frequencies is in agreement with the analysis of the lateral deformations which occurred in the experiments performed in space (Martínez private communication) in which the excitation seems to be of random type, although only the first mode of the lateral deformations $(m=1)$ could be detected because the sampling rate used reduces the measurable frequency range to $0-0.25 \mathrm{~Hz}$. Anyway, the first mode should be the best observed as it is the most amplified (figure 8).

The resonant frequencies, made dimensionless with the aid of (5.1) for the values corresponding to the liquid employed, are shown in figure 10 and table 2 . Note the good agreement with the theoretical results for the case of isolated liquid bridge. 


$\begin{array}{cccc}\text { Run } & A & \Phi_{\mathrm{R}} & \omega_{\mathrm{R}} \\ 2 & 2.71 & 0.18 & 0.57 \\ 3 & 2.71 & 0.19 & 0.59 \\ 4 & 2.86 & 0.17 & 0.54 \\ 5 & 2.71 & 0.17 & 0.54 \\ 6 & 2.57 & 0.19 & 0.59\end{array}$

TABLE 2. Slenderness $A$, resonant frequency $\Phi_{\mathrm{R}}(\mathrm{Hz}), \omega_{\mathrm{R}}$ dimensionless pulsation (5.1). Results from experiments with non-surrounded liquid bridges performed in the Spacelab-D1 Mission (Martínez private communication).

\section{Conclusions}

The linear non-axisymmetric oscillations of a liquid bridge have been considered. The main characteristics of the dynamics have been described: free frequencies and deformation modes, velocity field and the influence of a surrounding immiscible liquid.

The non-axisymmetric oscillations do not present stability changes, they are always stable. The threshold required to excite an azimuthal mode $m$ increases as $m$ increases.

The symmetric modes (with respect to the plane $z=0$ ) have an important bidimensional $((r-\theta)$-plane) flow component, whereas antisymmetric modes are essentially three-dimensional.

The influence of an outer liquid is the same as the axisymmetric case: in the interface shapes it is negligible whereas free frequencies are reduced.

An experimental study has been performed by using the neutral buoyancy technique, in order to check the resonant frequencies. The method is based on the determination of the transfer function of the response of the interface deformation to a lateral excitation by the frequency-sweep technique. Only the case $m=1$ has been considered because of the difficulties of exciting higher-order modes. Results appear to be in good agreement with the results predicted for isodense liquids.

The influence of the excitation amplitude, even as large as $0.25 R$, seems to be small, although deformation amplitudes up to $0.75 R$ have been produced, so that the linear model seems to be valid for a range larger than expected.

The phase of the maximum deformation behaves as in the case of a slightly damped system, showing approximately a $\frac{1}{2} \pi$ delay with respect to the displacement of the excitation disk.

Other experimental data, extracted from the behaviour of non-surrounded liquid bridges observed during the experiments performed in space, compared well with the appropriate theoretical results $(\rho=0)$.

Concerning viscosity effects the condition for considering unsteady flows as inviscid is that the viscous terms are negligible compared with the inertial ones, that is $S t R e=\lambda^{2} \Omega / \nu \gg 1$ (Levich 1962; Sanz 1985), where $S t, R e, \lambda, \Omega$ and $\nu$ are the Strouhal and Reynolds numbers, the wavelength $(L / N)$, the dimensional pulsation $\Omega=\left(\sigma /\left(\rho^{\mathrm{i}} R^{3}\right)\right)^{\frac{1}{2}} \omega$ and the kinematic viscosity. This condition can be rewritten in the form $\lambda \gg l_{b}=(v / \Omega)^{\frac{1}{2}}$ which is the penetration depth inside a fluid of the oscillation of an infinite plate (Stokes' second problem), so that the wavelength should be much larger than the viscous penetration of the oscillation. Rearranging, one finds St $R e=4(\Lambda / N)^{2} \omega / O h$, where $O h=\nu\left(\rho^{1} /(\sigma R)\right)^{\frac{1}{2}}$ is the Ohnesorge number. As can be deduced from the expression above, viscous effects increase as the pulsation $\omega$ and/or 
the wavelength $A / N$ decrease. But for a given $A, \omega$ and $N$ are not uncoupled, increasing the excitation frequency leads to the oscillation mode $N$ increasing, the combined effect of both factors in $S t R e$ is $\omega / N^{2}$, so that $S t R e$ decreases and thus viscous effects become larger. Thus, only the lower modes ( $N$ small) can be experimentally obtained. Actually, in experiments viscous effects prevent one from obtaining the upper ones, as the oscillatory motion of the disks only penetrates a few wavelengths into the liquid bridge. For the values of the parameters of the neutral buoyancy experiment configuration here employed one obtains $S t R e \approx(10 \Lambda / N)^{2} \omega$, the lowest value reached being $S t R e \approx 100$ for the case $A=2.6, N=4, \omega=2.5$. In the case of Spacelab D-1 experiments (table 2), StRe $\approx(15 \Lambda / N)^{2} \omega$ so that St $R e \approx 800$.

We wish to thank Drs J. Meseguer and I. Martínez for helpful discussions.

\section{Appendix}

$$
\left.\begin{array}{c}
s_{m 0}^{2}=\frac{m k_{m 0}}{S_{m 0}}, \quad s_{m n}^{2}=\frac{l_{n} k_{m n} I_{m}^{\prime}}{S_{m n} I_{m}} \\
S_{m 0}=1+\rho \frac{B^{2 m}+1}{B^{2 m}-1}, \quad S_{m n}=1+\rho\left[\frac{K_{m}\left(l_{n}\right)}{I_{m}\left(l_{n}\right)}-\frac{K_{m}^{\prime}\left(l_{n} B\right)}{I_{m}^{\prime}\left(l_{n} B\right.}\right]\left[-\frac{K_{m}^{\prime}\left(l_{n}\right)}{I_{m}^{\prime}\left(l_{n}\right)}+\frac{K_{m}^{\prime}\left(l_{n} B\right)}{I_{m}^{\prime}\left(l_{n} B\right)}\right]^{-1} \\
C_{10}=1, \quad k_{10}=6 \Lambda^{-2}, \quad C_{12 p}=-\frac{2 s^{2}}{s_{10}^{2}-s^{2}} \frac{k_{10}}{k_{12 p}}, \quad C_{12 p-1}=2\left(\Lambda k_{12 p-1}\right)^{-1} \\
C_{m 0}=-\frac{1}{2} \gamma_{m} \sinh \left(\gamma_{m} \Lambda\right) D_{m 0}, \quad C_{m 2 p}=-\gamma_{m} \sinh \left(\gamma_{m} \Lambda\right) D_{m 2 p}, \\
C_{m 2 p-1}=-\gamma_{m} \cosh \left(\gamma_{m} \Lambda\right) D_{m 2 p-1} \\
z=\sum_{p=1}^{\infty} D_{12 p-1} \cos l_{2 p-1}(z+\Lambda), \quad D_{i j}=\frac{2}{\Lambda k_{i j}}, \\
\frac{1}{2} z^{2}=\frac{1}{6} \Lambda^{2}-\Lambda \sum_{p-1}^{\infty} D_{12 p} \cos l_{2 p}(z+\Lambda) \\
\cosh \left(\gamma_{m} z\right)=C_{m 0}+\sum_{p-1}^{\infty} C_{m 2 p} \cos l_{2 p}(z+\Lambda) \\
\sinh \left(\gamma_{m} z\right)=\sum_{p=1}^{\infty} C_{m 2 p-1} \cos l_{2 p-1}(z+\Lambda) \\
C_{02 p}=D_{02 p} \sin \Lambda, \quad C_{02 p-1}=D_{02 p-1} \cos \Lambda
\end{array}\right\}
$$

\section{REFERENCES}

BAUER, H. F. 1982 Coupled oscillations of a solidly rotating liquid bridge. Acta Astron. 9, 547-563.

Bendat, J. S. \& Piersol, A. G. 1971 Random Data: Analysis and Measurement Procedures. WileyInterscience.

Bisch, C., LaSeK, A. \& Rodot, H. 1982 Comportement hydrodynamique de volumes liquides spheriques semi-libres en apesanteur simulée. J. Méch. Théor. Appl. 1, 165-183.

Elagin, M. P., Lebedev, A. P. \& Tsmelev, A. V. 1982 Laboratory modeling of the stability and dynamics of free liquid zones. In Hydromechanics and Heat and Mass Transfer in Zero-Gravity (in Russian), pp. 24-33. Moscow: Nauka. 
Entov, V. M. \& Yarin, A. L. 1984 The dynamics of liquid jets in air. J. Fluid Mech. 140, 91-111.

Fowle, A. A., Wang, C. A. \& Strong, P. F. 1979 Experiments on the stability of conical and cylindrical liquid columns at low Bond numbers. ADL Ref. C-82435, Arthur D. Little.

LEE, H. C. 1974 Drop formation in a liquid jet. IBM J. Res. Dev. 18, 364-369.

Levich, G. L. 1962 Physicochemical Hydrodynamics, p. 592. Prentice-Hall.

MARTínEZ, I. 1978 Floating zone. Equilibrium shapes and stability criteria. In COSPAR Space Research, vol. 18, pp. 519 522. Pergamon.

Martínez, I. 1987 a Stability of liquid bridges. Results of SL-D1 experiment. Acta Astron. 8, 449-453.

Martínez, I. $1987 b$ Stability of long liquid columns in Spacelab D-1. ESA SP-256, pp. 235-240.

Martínkz, I. \& Rivas, D. 1982 Plateau tank facility for simulation of Spacelab experiments. Acta Astron. 9, 339-342.

Mesequer, J. 1983 The breaking of axisymmetric slender liquid bridges. J. Fluid Mech. 130, $123-151$.

Meseguer, J., Mayo, L. A., Llorente, J. C. \& Fernández, A. 1985 Experiments with liquid bridges in simulated microgravity. J. Cryst. Growth 73, 609621.

Meseguer, J. \& SANZ, A. 1985 Numerical and experimental study of the dynamics of axisymmetric liquid bridges. J. Fluid Mech. 153, 83-101.

Meseguer, J. \& Sanz, A. 1987 Oscilaciones de puentes liquidos en el Spacelab D1. Anales RSE Física 1, 57-68.

Meseguer, J., Sanz, A. \& López, J. 1986 Liquid bridge breakages aboard Spacelab-D1. J. Cryst. Growth 78, 325 334 .

RAYLEIGH, LORD 1945 The Theory of Sound, vol. 2, pp. 351-355. Dover.

Rhone-Poulenc 1978 Rhodorsil silicones. Ref. X-03-03b. Rhone-Poulenc Chimie Fine, Department Silicones, Paris.

Rivas, D. \& Meseguer, J. 1984 One-dimensional, self-similar solution of the dynamies of axisymmetric slender liquid bridges. J. Fluid Mech. 138, 417-429.

Russo, M. J. \& STEEN, P. H. 1986 Instability of rotund capillary bridges to general disturbances: experiment and theory. J. Colloid Interface Sci. 113, 154-163.

SANZ, A. 1985 The influence of the outer bath in the dynamics of axisymmetric liquid bridges. J. Fluid Mech. 156, 101-140.

Sanz, A. \& Martínez, I. 1983 Minimum volume for a liquid bridge between equal disks. J. Colloid Interface Sci. 93, 235-240.

Struik, D. J. 1957 Classical Differential Geometry. Addison Wesley.

TомотікA, S. 1935 On the instability of a cylindrical thread of a viscous liquid suriounded by another viscous fluid. Proc. R. Soc. Lond. A 150, 322-337.

VeGa, J. M. \& Perales, J. M. 1983 Almost cylindrical isorotating liquid bridges for small Bond numbers. In Materials Science under Microgravity ESA SP-191, pp. 247-252. 